\title{
Many particle spectroscopy of atoms, molecules, clusters and surfaces: international conference MPS-2016*
}

\author{
Alexei N. Grum-Grzhimailo ${ }^{1}$, Yuri V. Popov ${ }^{1,2}$, Elena V. Gryzlova ${ }^{1}$, and Andrey V. Solov'yov ${ }^{3,4, a}$ \\ 1 Skobeltsyn Institute of Nuclear Physics, Lomonosov Moscow State University, Leninskie Gory 1, 119991 Moscow, Russia \\ 2 Joint Institute for Nuclear Research, Zholio-Kyuri str. 6, 141980 Dubna, Russia \\ 3 MBN Research Center, Altenhöferallee 3, 60438 Frankfurt am Main, Germany \\ 4 A.F. Ioffe Physical-Technical Institute, Politekhnicheskaya ul. 26, 194021 St. Petersburg, Russia
}

Received 20 April 2017

Published online 27 July 2017 - (C) EDP Sciences, Società Italiana di Fisica, Springer-Verlag 2017

\begin{abstract}
The conference on Many Particle Spectroscopy of Atoms, Molecules, Clusters and Surfaces (MPS-2016) brought together near to a hundred scientists in the field of electronic, photonic, atomic and molecular collisions, and spectroscopy from around the world. We deliver an Editorial of a topical issue presenting original research results from some of the participants on both experimental and theoretical studies involving many particle spectroscopy of atoms, molecules, clusters and surfaces.
\end{abstract}

The International Conference Many Particle Spectroscopy of Atoms, Molecules, Clusters and Surfaces (MPS-2016) was held at the Physics Department of the Lomonosov Moscow State University from August 23 to 26, 2016. This conference has a long-standing history going back to 1986 , when 44 (mostly European) scientists met at the Université Paris-Sud in Orsay. The conference called "Collisions (e,2e) et problemes connexes", and in total 17 talks were delivered. There it was decided to organize regular meetings devoted to the ionization processes in atoms. After a while, in 1989, the conference got supported through the European Science Program (Laboratory Twinning) that was focused on the cooperation of laboratories in Europe in the field of "Ionisation et spectroscopies de coincidence". The same year the conference has changed its name to "(e,2e) Collisions and Related Problems" and took place in Kaiserslautern (Germany). The conferences in Rome (Italy), Cambridge (UK), and Paris (France) followed after during the next three years. In 1994 the title of the conference has been changed to "Euroconference on Coincident Studies of Electron and Photon Impact Ionization". Under this title the conference took place in Colmar (France), Trieste (Italy), Belfast (UK) and other venues. After the conference in Belfast, the proceedings of the conference has been published or represented by special issues in different journals [1-3].

This research community participated in the major international conferences in the field, such as International Conference on Photonic, Electronic and Atomic Colli-

\footnotetext{
* Contribution to the Topical Issue "Many Particle Spectroscopy of Atoms, Molecules, Clusters and Surfaces", edited by A.N. Grum-Grzhimailo, E.V. Gryzlova, Yu.V. Popov, and A.V. Solov'yov.

a e-mail: solovyov@mbnresearch.com
}

sions (ICPEAC), and its satellites (e.g. International Symposium on (e,2e), Double Photoionization, and Related Topics; International Symposium on Polarization and Correlations in Electronic and Atomic Collisions).

Under the name Many-Particle Spectroscopy of Atoms, Molecules, Clusters, and Surfaces the conference was held in Halle, Germany in 2000. Kluwer Academic/ Plenum Publishers published nice proceedings edited by Berakdar and Kirschner [4]. However, the conference title was not finally fixed at that time and thus the following two meetings were called "International Conference on Electron and Photon Impact Ionization and Related Topics" (2002, Metz, France [5] and 2004, Louvain-la-Neuve, Belgium [6]). The current name was finally settled in 2006 at the conference in Rome, Italy [7]. Since then, the conference was held every two years between the biannual ICPEAC conferences [8-11] (unfortunately, the reports presented on the conference in Berlin were not published).

The MPS-2016 conference was held for the first time in Russia. Near to 100 participants from 20 countries gathered together to discuss the latest developments in the field of radiation interaction with matter. Topics of the conference were in line with the traditional directions of the MPS conferences of recent years. The scientific program of the conference included 37 invited talks and two sessions with poster presentations. It was focused on the actual experimental and theoretical problems of the interaction of radiation of different nature (electrons, photons, atoms, heavy charged particles) with various targets - atoms, molecules, clusters, surfaces of solids and nanostructures.

Studies of thin films and kinetics of plasma of various nature, being the traditional areas of application of collision spectroscopy methods, have also been presented at the conference. The wealth of new information 
about the processes in the universe supplied by space vehicles, the issues of the influence of space factors on biological tissues, development of the ion beam cancer therapy, the creation of new materials and nanostructures and their application in the design of products exposed to the flows of particles, as well as many more other current scientific challenges lead to the need for fundamental research, both experimental and theoretical, on collisions of charged particles and neutral atoms with matter.

$(\mathrm{e}, 2 \mathrm{e})$ : The processes of ionization are among the most discussed at the MPS conferences. They involve all sorts of ionization of atoms, molecules, clusters and solids either by electron or ion impact, or in an external electromagnetic or a laser field. Different topics have been a focus of the MPS community over the years. The so-called $(\mathrm{e}, 2 \mathrm{e})$ processes being among the main motivations for the creation of the MPS conference series were intensively studied towards the end of the last century. Mainly, two different kinematical cases were investigated: either a fast electron knocks out a slow electron, and then both are measured in coincidence, or two fast electrons, the scattered and the knocked-out, are measured with equal angles and energies. The former case, which is called a dipolar reaction, is characterized by a much larger cross section, so that it allows studying the dominant ionization mechanisms $[12,13]$. The latter case, the binary reaction, is usually employed for the Electron Momentum Spectroscopy (EMS) of the target, where one measures momentum distribution of the knocked-out electron in the target. Further information on EMS can be found in review papers [14-16] and in the book [17]. Later, even more sophisticated experiments of both kinds were performed for $(\mathrm{e}, 3 \mathrm{e})$ ionization $[13,15,18-20]$.

The reports on atomic ionization by $\gamma$ quanta have been on the agenda of the MPS conferences since their beginning in 1986, being actively discussed in the literature and during the conferences. The basics of the theoretical and experimental studies of these reactions can be found in the review papers $[21,22]$. There have been also studied, predominantly by the theoretical community, laser assisted (e,2e) reactions. Here again, the dipolar kinematics reactions [23] as well as binary ones [24] can be distinguished.

Hundreds of theoretical and experimental papers focusing on $(\mathrm{e}, 2 \mathrm{e})$ reactions have been already published, and their number still grows, although slower. Nowadays, both the projectile and the target are getting more and more varied, as well as experimental setups. More attention is paid to collisions of heavy atoms and molecules with cations, in particular, protons. The experiments are carried out on the modern COLTRIMS (Cold Target Recoil Ion Momentum Spectroscopy) setups [25-27].

Most of the above-mentioned (e,2e)-ionization-related topics are represented in this Topical Issue of European Physical Journal D devoted to the MPS-2016 conference. In paper [28], a single ionization process, $(\mathrm{e}, 2 \mathrm{e})$, of $\mathrm{Ar}$ and of few molecular targets: $\mathrm{CH}_{4}, \mathrm{NH}_{3}$ and $\mathrm{H}_{2} \mathrm{O}$, is considered. Several relatively recent models of Post Collision Interaction (PCI) are utilised. Significant differences are ob- served between these models and the experimental data. Paper [29] presents the model that includes correlations both in the initial state and in the final state, and its application to the calculations of the fivefold differential cross sections for double ionization of neon, (e,3e). The results of the model calculations are compared to the available experimental data performed at high incident energy. Disagreements between theory and experiment should be a subject of further theoretical investigations. Another work [30] discusses the properties of the two-electron continuum wave function that involve the electron-electron interaction. The idea of reported approach is to include the phase into a Convoluted Quasi Sturmian (CQS) basis whose elements already behave asymptotically as an outgoing (incoming) six-dimensional spherical wave aiming at constituting a suitable set of basis functions for the threebody Coulomb continuum wave function representation in the entire space for the numerical solution of the $(\mathrm{e}, 3 \mathrm{e})$ non-homogeneous Schrödinger equation in a two-channel case.

Paper [31] discusses the bond oscillation effect within the (e,2e) EMS kinematical conditions in the measured electron momentum distributions of $\mathrm{CCl}_{4}$ and $\mathrm{CF}_{4}$ and demonstrates that the period of the interference pattern reflects the internuclear distance between the constituent halogen atoms. The theoretical work [32] is devoted to $(\mathrm{e}, 2 \mathrm{e})$ studies of the $1 \mathrm{t}_{2}$ orbital of methane. The authors use a basis set expansion over the generalized Sturmian functions with correct asymptotic behavior. The results reported partially reproduce the experimentally observed cross sections features. Thus, for an incident electron energy $250 \mathrm{eV}$, ejected electron energy $30 \mathrm{eV}$ and a scattering angle $-20^{\circ}$, a double peak structure in the cross section binary region is predicted, which is a clear signature of the $p$-nature of the molecular orbital. Finally, paper [33] reports on the first theoretical analysis of the laser-assisted $(\mathrm{e}, 2 \mathrm{e})$ process on a $\mathrm{H}_{2}^{+}$molecular ion.

Concluding on $(e, 2 e)$, it is worth noting that most of the related papers presented in this topical issue are devoted to the reactions with molecules. Obviously the spotlight of scientific interest in the field moves in this direction.

Heavy particle collisions: Several papers in the topical issue are devoted to ionization by a bare projectile ion (proton). Single ionization from biological molecules is considered in [34]. In particular, the single differential and total cross sections for single ionization and single electron capture from biological targets, namely, water vapor and DNA nucleobase molecules, by bare projectile impact: $\mathrm{H}^{+}$, $\mathrm{He}^{2+}$, and $\mathrm{C}^{6+}$ are obtained. The calculations are performed within the Continuum Distorted Wave - Eikonal Initial State (CDW-EIS) approximation for the final state of the reaction. This study aims to obtain a reliable set of theoretical data to be used as input in Monte Carlo simulations for micro- and nano-dosimetry. Paper [35] reports on the theoretical study of the double ionization of helium by $6 \mathrm{MeV}$ proton impact by means of the three-body driven equation within the generalized Sturmian functions approach. The theoretical description provides a satisfactory 
reproduction of the measured data on a relative scale. The authors also formulate some predictions that have to stimulate further measurements. Paper [36] demonstrates that in COLTRIMS experiments on atomic ionization by fast ions, the effects of the target center-of-mass wave packet might be of greater importance than those of the projectile wave packet. This paper contributes to a long-time discussion about the role of coherence/incoherence of an ion-projectile beam.

The multiscale understanding of the underlying physics of radiation in living matter is an essential prerequisite of any research at the physics and biology frontier [37-39]. This topic was in the focus of the European Research over the last decade. Thus, during 2010-2014, the European Concerted Research Action, COST Action MP1002: nano-scale insights in ion beam cancer therapy (Nano-IBCT) was devoted to acquiring a deeper understanding of radiation-induced damage with ions on the nanoscopic and molecular level. The research conducted within the action by the broad international community was presented in the two topical issues of the European Physical Journal D [40,41] and in the book [37]. In this context, Monte Carlo simulations are now often utilised as a popular tool for investigating the radio-induced interactions. Paper [42] of the MPS-2016 topical issue presents a code for describing the track-structure of protons and its secondaries in living matter that includes both water and biological species. This quantum-mechanically based proton transport modeling in a "realistic" biological environment paves a way for further investigations focused on a water vs. DNA analysis via Monte Carlo simulations of proton transport in complex environment.

Collisions of ions with hydrocarbon molecular targets have been a subject of interest in both experimental and theoretical physics. Electron removal and fragmentation of the methane molecule induced by proton and antiproton impact are obtained theoretically in [43] in the broad impact energy range. Methane being the simplest hydrocarbon molecule $\mathrm{CH}_{4}$ is one of the interesting targets to study due to a large number of applications in different fields, such as astrophysical and atmospheric sciences. For example, a considerable fraction of the Titan's atmosphere consists of $\mathrm{CH}_{4}$ molecules that are continuously fragmented by impinging high-energy electrons, protons and atomic nuclei from the cosmic rays [44]. Data on the antiproton impact were obtained in [43] for the first time. Electron impact double ionization of methane is studied in another paper of this issue [45]. In particular, the influence of the molecular target orientation on the total double ionization cross sections and energetic distributions of the outgoing two electrons are obtained.

Photonics: Particular attention at the MPS-16 was paid to the quantum processes in electromagnetic fields of the new-generation light sources, to the interaction of radiation with nanoparticles and large molecules, to the quantum theory of processes in many-particle systems. The conference contributed to advances in such areas of fundamental research as the investigation of behavior of multiparticle systems and nano-objects under the influ- ence of intense X-ray radiation and the properties of nonlinear photoprocesses in this spectral range, the visualization of intramolecular dynamics at the atomic level, the development of new methods for studying the surfaces of solid materials, the creation and study of previously inaccessible exotic atomic and molecular states, and the development of new methods for modelling quantum processes.

The need of addressing the aforementioned fundamental problems at the MPS-2016 conference arose from many circumstances. Recently, sources of intense XUV and $\mathrm{X}$-ray radiation have been created and are being improved: free-electron lasers, higher-order laser harmonics generators, and new undulator lines. This development is of significant importance for the progress of quantum optics in the far UV and X-ray region, for applications in the quantum control of photoprocesses, for observations of reactions with femto- and attosecond time resolution, for holography at the atomic level, and for a number of other applications. It is necessary for a deep understanding of the dynamics of both photoprocesses in small quantum objects and more complex phenomena: the fundamental processes of chemistry and the production of new materials and drugs, the behavior of matter under extreme conditions and the reconstruction of the nuclear fusion process, the visualization of individual biomolecules or their nanocrystals, and new ways of generating radiation.

Paper [46], related to traditional spectroscopy of energy levels, presents a newly developed approach for accurate calculation of atomic structure based on a modified configuration interaction + many-body perturbation theory (CI+MBPT) method, which was applied to the analysis of the $\mathrm{Si}$ atom. The calculated energies significantly improve the previous theoretical results [47].

Paper [48] describes an experiment to measure the effective cross section for the electron excitation of the $\mathrm{Pb}^{+}$resonance $143.4 \mathrm{~nm}$ line, in the near threshold energy region. These are the first data regarding the excitation cross-sections at the interaction of electrons with $\mathrm{Pb}^{+}$ ions. The research builds on previous publications of the same group using a crossed-beam technique. Considerable structure is seen in the cross section, which is attributed to cascade decays of excited and autoionizing states. The data obtained are essential for many applications including diagnostics of emitting plasma and abundances of elements within the plasma, research of atomic structure and testing theories, applications in astrophysics. In particular, the $\lambda=143.4 \mathrm{~nm}$ line was detected in the emission spectra of several stars $[49,50]$.

The rapidly developing field of interaction of X-ray free electron laser (FEL) radiation with matter is represented by two papers $[51,52]$. In view of the recent breakthrough in generating time-coherent two-color phase-shifted extreme ultraviolet (XUV) pulses at FEL Fermi (Trieste, Italy) and future applications [53,54], a new theoretical investigation [51] on the coherent control of atomic systems in the extreme ultraviolet (XUV) energy range is timely, continuing first theoretical predictions $[55,56]$. The control of the angular distribution of photoelectrons is achieved by bichromatic pulse whose fundamental frequency is tuned 
near an atomic state of neon. Another study [52] has been prepared on the basis of experiments performed at the SACLA XFEL Laser Facility (Japan) in a collaboration involving universities and laboratories from Japan and Russia [57]. A simple theoretical recipe is proposed to estimate the size of the secondary electron cloud, generated in matter by incoming hard X-ray photons. An exclusive response of the LiF crystal to deposited X-ray doses by proportional generation of secondary electrons, which cause creation of color centres density inside the crystal, provides a unique possibility to validate the theoretical predictions for the size of the electron cloud with submicron resolution. The findings may have wide practical interest and could be useful for further development in many scientific and industrial areas, including laser and atomic physics, X-ray science and technology, high energy density science, X-ray optics, the creation and application of intense X-ray sources, biology and medicine.

Interaction of the laser radiation with atomic and molecular systems is one of traditional topics at the MPS conferences. Besides already mentioned work [33], this issue contains other articles in this field [58-60]. Two of them develop methods of solution of the time-dependent Schrödinger equation (TDSE), which is currently a very important field of theory in view of applications to interaction of short radiation pulses with matter. A new approach to solving the TDSE for a particle in a strong laser field within a Faddeev-like formalism is tested in [58]. The approach is based on a new series expansion of two coupled integral equations. Contrary to the series in power of the Coulomb binding potential, these new perturbative terms include successively both intermediate Coulomb and Volkov Green's functions. Calculations show that the results rapidly converge towards those obtained by explicit numerical solution of the TDSE, at least at not low frequencies. Thus the method promises good perspectives. In [59] the same group uses separable potential method and performs very extensive analysis of the results for ionization of hydrogen atom by strong low-frequency field. In this method, the TDSE in the momentum space reduces to a set of coupled one-dimensional linear Volterra integral equations. In particular, the conventional strong-field approximation (SFA) turns out to be a limiting case of the method. Despite the fact that different approaches have been developed for treating hydrogen ionization in strong field regime, the authors managed to bring novelty into this generally well explored field.

Nanosystems and beyond: A bunch of topics that are traditionally presented at the MPS conferences are related to the studies of finite systems, such as atomic and molecular clusters, thin films, nanostructured materials, e.g. fullerenes or carbon nanotubes, or surface structures. Properties of these systems are often rather unique, differ from both the properties of single atoms or molecules and the solid state, and typically are influenced by the presence of boundaries within the system. Modern experimental techniques have made it possible to discover and study such systems in details. By increasing the system size, one can observe the emergence of the physical fea- tures in the system, such as plasmon excitations, electron conduction band formation, superconductivity and superfluidity, phase transitions, fission and many more. Most of these many-body phenomena exist in solid state but are absent for single atoms.

The aforementioned systems provide a "laboratory" in which the major interactions and many-body effects present also in solids can be analysed and studied as a function of their size. The systems also straddle the limit between microscopic and quasi-classical systems, so they can be used to probe the boundary between quantum mechanics and semi-classical systems. They also become the appropriate physical objects for studying statistical and thermodynamic laws in nanoscale systems, both classical and quantum, and are tractable computationally (up to the nanoscale) by ab initio methods. It is important that most of the systems can be made and observed in the laboratory by using modern beam, collision or deposition techniques. They provide new examples of many-body forces in regimes which is different from those of atomic, nuclear or solid-state physics, although related to all of them. Such systems can serve as building blocks for new forms of matter, e.g. the formation of cluster-based molecules and new materials.

Beyond MPS, these topics are widely discussed also at other international conferences by the adjacent research communities. Here, let us mention the key international meetings in this research area. A biannual series of International Symposia on Atomic Cluster Collisions $(\text { ISACC })^{1}[61-64]$ is devoted to the structure, properties and dynamics of complex nuclear, atomic, molecular, cluster, nanoscopic and biological systems studied primarily by means of photonic, electronic, heavy particle and atomic collisions. The biannual International Conferences on Dynamics of Systems on the Nanoscale (DySoN) $[65,66]$ promote the growth and exchange of interdisciplinary scientific information on the structure formation and dynamics of animate and inanimate matter on the nanometre scale.

This research area is represented in the current topical issue by the work [67] discussing He ion irradiation effects on multiwalled carbon nanotubes (MWCNT) structure. It was demonstrated that as the fluence increases, initially the MWCNTs show disorder due to the produced defects, and then amorphization under still higher fluence of ion irradiation. Thermal and athermal mechanisms of the radiation induced MWCNTs modifications are discussed.

Damage of porous organosilicate glass (OSG) films with low dielectric constants (low- $k$ films) in plasma processing is a critical problem for modern microelectronics. For this problem, the understanding and revealing of the basic reaction steps for radicals etching and damage are of importance. The manuscript [68] reports on the experimental and DFT studies of nitrogen atoms interactions with $\mathrm{SiOCH}$ low- $k$ films. The effects of $\mathrm{N}$ atoms on OSG films are studied both experimentally by Fourier Transform InfraRed (FTIR) spectroscopy method and theoretically by the density functional theory (DFT)

\footnotetext{
1 http://www.isacc-portal.org/
} 
method. Another work [69] discusses the interaction of fluorine atoms with OGS films reporting on both the experimental observations and the results of DFT simulations. The one-dimensional 1-D Monte Carlo and gassurface kinetics model, as well as the density functional theory, have been used for studying the multi-step mechanism of OSG films damage and etching. The results of simulations are verified through the FTIR spectroscopy data. The photoelectric properties of the pyrolitic CdS films and Li-doped are investigated in [70] where the effect of photocurrent limiting is reported. The effect is interpreted by the surface-barrier mechanism of the current transfer, and the theoretical dependencies of mobility, conductivity and photocurrent on the modulation of the potential barrier under photoexcitation are obtained. The analysis of experimental dependencies of photocurrent on the excitation intensity in the context of the aforementioned theoretical advances is performed.

An invaluable help of the "CTO Congress" Group, and support of Physical Faculty and Skobeltsyn Institute of Nuclear Physics of M.V. Lomonosov Moscow State University in organization of the MPS-2016 is greatly acknowledged.

\section{Author contribution statement}

All the authors contributed equally in writing this Editorial.

\section{References}

1. C.T. Whelan, H.R.J. Walters, A. Lahmam-Bennani, H. Ehrhardt (eds.), (e,2e) and Related Topics, NATO ASI Series C: Mathematical and Physical Sciences (Kluwer Acad. Publ., Dordrecht, 1993), Vol. 414

2. C.T. Whelan, H.R.J. Walters (eds.), Coinicidence Studies of Electron and Photon Impact Ionization (Plenum Publ. Corp., 1997)

3. L. Langlois, G. Nguen Vien, A. Pochat, S. Rioual, O. Robaux, B. Rouvellou, R.J. Tweed (eds.), in Proceedings of the International Conference on Coincidence Spectroscopy, Brest, France, 1998, J. Phys. IV France 9 (Pr6) (1999)

4. J. Berakdar, J. Kirschner (eds.), Many-Particle Spectroscopy of Atoms, Molecules, Clusters and Surfaces (Kluwer Academic, Plenum Publishers, New York, 2001)

5. L.U. Ancarani (ed.), in Proceedings of the Int. Conf., Electron and Photon Impact Ionisation and Related Topics 2002, Metz, France, IOP Conf. Ser. (IOP, Bristol, 2003), Vol. 172

6. B. Piraux (ed.), in Proceedings of the International Conference on Electron and Photon Impact Ionization and Related Topics 2004, Louvain-la-Neuve, Belgium, IOP Conf. Ser. (IOP, Bristol, 2005), Vol. 183

7. G. Stefani, L. Avaldi (eds.), in International Conference on Many Particle Spectroscopy of Atoms, Molecules, Clusters and Surfaces, Rome, 2006, J. Electron Spectr. Rel. Phenom. (2007), Vol. 161

8. D. Dowek, A. Lahmam-Bennani, P. Lablanquie, A. Maquet (eds.), in International Conference on Many Particle Spectroscopy of Atoms, Molecules, Clusters and Surfaces, Paris, 2008, J. Phys.: Conf. Ser. (2008), Vol. 141
9. M. Takahashi, K. Ueda (eds.), in International Conference on Many Particle Spectroscopy of Atoms, Molecules, Clusters and Surfaces, Tohoku University, Sendai, 2010, J. Phys.: Conf. Ser. (2011), Vol. 288

10. A. Wobst, T. Lischke, B. Langer, A. Dorn, U. Becker, in International Conference on Many Particle Spectroscopy of Atoms, Clusters and Surfaces, Berlin, 2012

11. L.U. Ancarani (ed.), in International Conference on Many Particle Spectroscopy of Atoms, Molecules, Clusters and Surfaces, Metz, 2014, J. Phys.: Conf. Ser. (2015), Vol. 601

12. H. Ehrhardt, K. Jung, G. Knoth, P. Schlemmer, Z. Phys. D 1, 3 (1986)

13. A. Lahmam-Bennani, J. Phys. B 24, 2401 (1991)

14. M.A. Coplan, J.H. Moore, J.P. Doering, Rev. Mod. Phys. 66, 985 (1994)

15. V.G. Neudachin, Yu.V. Popov, Yu.F. Smirnov, Phys. Usp. 42, 1017 (1999)

16. Yu.V. Popov, O. Chuluunbaatar, V.L. Shablov, K.A. Kouzakov, Phys. Part. Nucl. 41, 543 (2010)

17. E. Weigold, I.E. McCarthy, Electron Momentum Spectroscopy (Kluwer Acad. Publ., New York, 1999)

18. N. Watanabe, Y. Khajuria, M. Takahashi, Y. Udagawa, P.S. Vinitsky, Yu.V. Popov, O. Chuluunbaatar, K.A. Kouzakov, Phys. Rev. A 72, 032705 (2005)

19. N. Watanabe, K.A. Kouzakov, Yu.V. Popov, M. Takahashi, Phys. Rev. A 77, 032725 (2008)

20. J. Berakdar, A. Lahmam-Bennani, C. Dal Cappello, Phys. Rep. 374, 91 (2003)

21. J.S. Briggs, V. Schmidt, J. Phys. B 33, R1 (2000)

22. J. Berakdar, H. Klar, Phys. Rep. 340, 473 (2001)

23. D. Khalil, A. Maquet, R. Taïeb, C.J. Joachain, A. Makhoute, Phys. Rev. A 56, 4918 (1997)

24. K.A. Kouzakov, Yu.V. Popov, M. Takahashi, Phys. Rev. A 82, 023410 (2010)

25. J. Ullrich, R. Moshammer, R. Dörner, O. Jagutzki, V. Mergel, H. Schmidt-Böcking, L. Spielberger, J. Phys. B 30, 2917 (1997)

26. R. Dörner, V. Mergel, O. Jagutzki, L. Spielberger, J. Ullrich, R. Moshammer, H. Schmidt-Böcking, Phys. Rep. 330, 95 (2000)

27. J. Ullrich, R. Moshammer, A. Dorn, R. Dörner, L.Ph.H. Schmidt, H. Schmidt-Böcking, Rep. Prog. Phys. 66, 1463 (2003)

28. S. Houamer, M. Chinoune, C. Dal Cappello, Eur. Phys. J. D 71, 17 (2017)

29. I. Kada, C. Dal Cappello, A. Mansouri, Eur. Phys. J. D 71, 41 (2017)

30. A.S. Zaytsev, L.U. Ancarani, S.A. Zaytsev, Eur. Phys. J. D 71, $177(2017)$

31. N. Watanabe, K. Katafuchi, M. Yamazaki, M. Takahashi, Eur. Phys. J. D 70, 268 (2016)

32. C.M. Granados-Castro, L.U. Ancarani, Eur. Phys. J. D 71, 65 (2017)

33. A.A. Bulychev, K.A. Kouzakov, Eur. Phys. J. D 71, 23 (2017)

34. M.A. Quinto, J.M. Monti, P.D. Montenegro, O.A. Fojón, C. Champion, R.D. Rivarola, Eur. Phys. J. D 71, 35 (2017)

35. M.J. Ambrosio, L.U. Ancarani, A.I. Gómez, G. Gasaneo, D.M. Mitnik, Eur. Phys. J. D 71, 127 (2017)

36. K.A. Kouzakov, Eur. Phys. J. D 71, 63 (2017)

37. A.V. Solov'yov (ed.), Nanoscale Insights into Ion-Beam Cancer Therapy (Springer International Publishing, 2017) 
38. A.V. Solov'yov, E. Surdutovich, E. Scifoni, I. Mishustin, W. Greiner, Phys. Rev. E 79, 011909 (2009)

39. E. Surdutovich, A.V. Solov'yov, Eur. Phys. J. D 68, 353 (2014)

40. M.A. Smialek, P. Limao-Vieira, N.J. Mason, A.V. Solov'yov, Eur. Phys. J. D 68, 312 (2014)

41. E. Surdutovich, G. Garcia, N.J. Mason, A.V. Solov'yov, Eur. Phys. J. D 70, 86 (2016)

42. M.A. Quinto, J.M. Monti, Ph.F. Weck, O.A. Fojón, J. Hanssen, R.D. Rivarola, Ph. Senot, C. Champion, Eur. Phys. J. D 71, 130 (2017)

43. A. Salehzadeh, T. Kirchner, Eur. Phys. J. D 71, 66 (2017)

44. T.E. Cravens, C.J. Lindgren, S.A. Levina, Planet. Space Sci. 46, 1193 (1998)

45. D. Oubaziz, Z. Aitelhadjali, M.A. Quinto, R. Boulifa, C. Champion, Eur. Phys. J. D 71, 160 (2017)

46. R.T. Imanbaeva, M.G. Kozlov, E.A. Konovalova, Eur. Phys. J. D 71, 115 (2017)

47. I.M. Savukov, Phys. Rev. A 91, 022514 (2015)

48. A.N. Gomonai, Yu.I. Hutych, A.I. Gomonai, Eur. Phys. J. D 71, 115 (2017)

49. J.A. Cardelli, S.R. Federman, D.L. Lambert, C.E. Theodosiou, Astrophys. J. 416, L41 (1993)

50. D.E. Welty, L.M. Hobbs, J.T. Lauroesch, D.C. Morton, D.C. York, Astrophys. J. 449, L1 (1995)

51. N. Douguet, E.V. Gryzlova, E.I. Staroselskaya, K. Bartschat, A.N. Grum-Grzhimailo, Eur. Phys. J. D 71, 105 (2017)

52. A.N. Grum-Grzhimailo, T. Pikuz, A. Faenov, T. Matsuoka, N. Ozaki, B. Albertazzi, S. Pikuz, Y. Inubushi, M. Yabashi, K. Tono, H. Yumoto, H. Ohashi, T. Ishikawa, R. Kodama, Eur. Phys. J. D 71, 69 (2017)

53. K.C. Prince, E. Allaria, C. Callegari, R. Cucini, G. De Ninno, S. Di Mitri, B. Diviacco, E. Ferrari, P. Finetti, D. Gauthier, L. Giannessi, N. Mahne, G. Penco, O. Plekan, L. Raimondi, P. Rebernik, E. Roussel, C. Svetina, M. Trovò, M. Zangrando, M. Negro, P. Carpeggiani, M. Reduzzi, G. Sansone, A.N. Grum-Grzhimailo, E.V. Gryzlova, S.I. Strakhova, K. Bartschat, N. Douguet, J. Venzke, D. Iablonskyi, Y. Kumagai, T. Takanashi, K. Ueda, A. Fischer, M. Coreno, F. Stienkemeier, Y. Ovcharenko, T. Mazza, M. Meyer, Nat. Photon. 10, 176 (2016)
54. N. Hartmann, J.M. Glownia, Nat. Photon. 10, 148 (2016) 55. A.N. Grum-Grzhimailo, E.V. Gryzlova, E.I. Staroselskaya, J. Venzke, K. Bartschat, Phys. Rev. A 91, 063418 (2015)

56. N. Douguet, A.N. Grum-Grzhimailo, E.V. Gryzlova, E.I Staroselskaya, J. Venzke, K. Bartschat, Phys. Rev. A 93, $033402(2016)$

57. T. Pikuz, A. Faenov, T. Matsuoka, S. Matsuyama, K. Yamauchi, N. Ozaki, B. Albertazzi, Y. Inubushi, M. Yabashi, K. Tono, Y. Sato, H. Yumoto, H. Ohashi, S. Pikuz, A.N. Grum-Grzhimailo, M. Nishikino, T. Kawachi, T. Ishikawa, R. Kodama, Sci. Rep. 5, 17713 (2015)

58. Yu. Popov, A. Galstyan, F. Mota-Furtado, P.F. O'Mahony, B. Piraux, Eur. Phys. J. D 71, 93 (2017)

59. A. Galstyan, Yu.V. Popov, F. Mota-Furtado, P.F. O'Mahony, N. Janssens, S.D. Jenkins, O. Chuluunbaatar, B. Piraux, Eur. Phys. J. D 71, 97 (2017)

60. S.N. Balybin, P.R. Sharapova, O.V. Tikhonova, Eur. Phys. J. D 71, 109 (2017)

61. J.-P. Connerade, A.V. Solovyov (eds.), Latest Advances in Atomic Clusters Collision: Fission, Fusion, Electron, Ion and Photon Impact (Imperial College Press, London, 2004)

62. J.-P. Connerade, A.V. Solovyov (eds.), Latest Advances in Atomic Clusters Collision: Structure and Dynamics from the Nuclear to the Biological Scale (Imperial College Press, London, 2008)

63. A.V. Korol, A.V. Solovyov, Eur. Phys. J. D 67, 1 (2013)

64. R. Prosmiti, P. Villarreal, G. Delgado-Barrio, A.V. Solov'yov, Eur. Phys. J. D 71, 36 (2017)

65. A.V. Solov'yov, J. Phys.: Conf. Ser. 438, 011011 (2013)

66. A.V. Korol, A.V. Solov'yov (eds.), Dynamics of Systems at the Nanoscale, Eur. Phys. J. D (2017), Vol. 71

67. E.M. Elsehly, N.G. Chechenin, A.V. Makunin, A.A. Shemukhin, H.A. Motaweh, Eur. Phys. J. D 71, 79 (2017)

68. E.N. Voronina, Yu.A. Mankelevich, T.V. Rakhimova, A.P. Palov, D.V. Lopaev, S.M. Zyryanov, A.I. Zotovich, M.R. Baklanov, Eur. Phys. J. D 71, 111 (2017)

69. Yu.A. Mankelevich, E.N. Voronina, T.V. Rakhimova, A.P. Palov, D.V. Lopaev, S.M. Zyryanov, M.R. Baklanov, Eur. Phys. J. D 71, 126 (2017)

70. T. Mayorova, V. Klyuev, A. Zvyagin, Eur. Phys. J. D 71, $43(2017)$ 